\title{
A Randomized, Open-Label, Phase II Study Comparing Pemetrexed Plus Cisplatin Followed by Maintenance Pemetrexed versus Pemetrexed Alone in Patients with Epidermal Growth Factor Receptor (EGFR)-Mutant Non-small Cell Lung Cancer after Failure of First-Line EGFR Tyrosine Kinase Inhibitor: KCSG-LU12-13
}

\author{
Kwai Han Yoo, MD ${ }^{1,2}$ \\ Su Jin Lee, MD, $P h D^{1}$ \\ Jinhyun Cho, MD ${ }^{3}$ \\ Ki Hyeong Lee, MD, PhD \\ Keon Uk Park, MD, PhD \\ Ki Hwan Kim, MD, PhD ${ }^{6}$ \\ Eun Kyung Cho, MD, PhD² \\ Yoon Hee Choi, MD, PhD ${ }^{7}$ \\ Hye Ryun Kim, MD, PhD ${ }^{8}$ \\ Hoon-Gu Kim, MD, PhD ${ }^{9}$ \\ Heui June Ahn, MD, PhD ${ }^{10}$ \\ Ha Yeon Lee, MD, $\mathrm{PhD}{ }^{11}$ \\ Hwan Jung Yun, MD, PhD ${ }^{12}$ \\ Jin-Hyoung Kang, MD, $\mathrm{PhD}^{13}$ \\ Jaeheon Jeong, MD, $P h D^{14}$ \\ Moon Young Choi, MD, PhD ${ }^{15}$ \\ Sin-Ho Jung, PhD' ${ }^{16}$ \\ Jong-Mu Sun, MD, PhD 1 \\ Se-Hoon Lee, MD, PhD ${ }^{1}$ \\ Jin Seok Ahn, MD, PhD ${ }^{1}$ \\ Keunchil Park, MD, PhD' \\ Myung-Ju Ahn, MD, PhD ${ }^{1}$
}

*A list author's affiliations appears at the end of the paper.

\section{Purpose}

The optimal cytotoxic regimens have not been established for patients with non-small cell lung cancer (NSCLC) who develop disease progression on first-line epidermal growth factor receptor tyrosine kinase inhibitor (EGFR-TKI).

\section{Materials and Methods}

We conducted a multi-center randomized phase II trial to compare the clinical outcomes between pemetrexed plus cisplatin combination therapy followed by maintenance pemetrexed $(\mathrm{PC})$ and pemetrexed monotherapy $(\mathrm{P})$ after failure of first-line EGFR-TKI. The primary objective was progression-free survival (PFS), and secondary objectives included overall response rate (ORR), overall survival (OS), health-related quality of life (HRQOL), and safety and toxicity profiles.

\section{Results}

A total of 96 patients were randomized, and 91 patients were treated at 14 centers in Korea. The ORR was $34.8 \%(16 / 46)$ for the PC arm and $17.8 \%(8 / 45)$ for the $P$ arm $(p=0.066)$. With 23.4 months of follow-up, the median PFS was 5.4 months in the PC arm and 6.4 months in the $P$ arm $(p=0.114)$. The median OS was 17.9 months and 15.7 months in PC and $P$ arms, respectively ( $p=0.787$ ). Adverse events $\geq$ grade 3 were reported in 12 patients (26.1\%) in the PC arm and nine patients $(20.0 \%)$ in the P arm $(p=0.491)$. The overall time trends of HRQOL were not significantly different between the two arms.

\section{Conclusion}

The outcomes of pemetrexed therapy in NSCLC patients with disease progression after firstline EGFR-TKI might not be improved by adding cisplatin.

\footnotetext{
Correspondence: Myung-Ju Ahn, MD, PhD Division of Hematology and Oncology, Department of Medicine, Samsung Medical Center, Sungkyunkwan University School of Medicine, 81 Irwon-ro, Gangnam-gu, Seoul 06351, Korea Tel: 82-2-3410-3438

Fax: 82-2-3410-1754

E-mail: silkahn@skku.edu

Key words

Non-small cell lung carcinoma, Epidermal growth factor receptor, Mutation, Pemetrexed, Cisplatin, Quality of life 


\section{Introduction}

Somatic mutations in the epidermal growth factor receptor (EGFR) gene account for about $30 \%-40 \%$ of non-small cell lung cancers (NSCLCs) in Asian patients and 10\%-15\% of NSCLCs in Caucasian patients [1,2]. The introduction of firstgeneration EGFR tyrosine kinase inhibitors (TKIs; gefitinib, erlotinib) and second-generation TKIs (afatinib) for the treatment of NSCLC patients with a sensitizing EGFR mutation has demonstrated a high response rate and prolonged progression-free survival (PFS) compared with platinum doublet as first-line therapy [3-5].

However, almost all patients eventually develop acquired resistance to EGFR-TKIs, with a median PFS of 9-14 months [6-8]. A randomized phase III trial comparing pemetrexed/ cisplatin/gefitinib with pemetrexed/cisplatin/placebo did not show improvement of clinical outcome in patients who showed disease progression on EGFR-TKI; therefore, platinum doublet chemotherapy is considered the standard treatment for this patient population [9]. Recently, a thirdgeneration EGFR-TKI (osimertinib) demonstrated significant improvement in response rate and PFS compared with platinum doublet chemotherapy in patients harboring the T79$0 \mathrm{M}$ mutation, which is the most common resistance mechanism to first- or second-generation EGFR-TKIs [10]. However, approximately $40 \%-60 \%$ of patients who develop resistance to EGFR-TKIs still need chemotherapy. Although platinum doublet chemotherapy is recommended as the treatment of choice in those patients, few data exist regarding the use of conventional chemotherapy for second-line treatment after failure of first-line EGFR-TKIs. D'Addario et al. [11] reported a $34.1 \%$ response rate and PFS of 6.8 months for a gemcitabine plus cisplatin regimen in NSCLC patients after failure of gefitinib. Wu et al. [12] performed retrospective analysis of second-line treatments after first-line gefitinib therapy and showed that platinum-based combination chemotherapy was associated with better PFS and overall survival (OS) than non-platinum regimens containing taxane or erlotinib.

Pemetrexed, a multi-target anti-folate agent, has been approved as second-line therapy regardless of first-line therapy (chemotherapy or targeted agents) in Korea, and only single-agent pemetrexed is available for patients who fail EGFR-TKIs. Our recent retrospective analysis showed that a combination of pemetrexed plus platinum had a better disease control rate than pemetrexed alone, suggesting the benefit of platinum doublets after failure of first-line EGFR-TKIs [13]. Moreover, the role of single-agent pemetrexed in this patient population has not been fully evaluated.

Based on these data, we conducted a prospective randomized phase II trial to compare the clinical outcomes between pemetrexed plus cisplatin combination therapy followed by maintenance pemetrexed and pemetrexed monotherapy in patients with EGFR-mutant NSCLC that progressed after first-line treatment with EGFR-TKIs.

\section{Materials and Methods}

\section{Patient eligibility}

We recruited patients who were at least 20 years old and had Eastern Cooperative Oncology Group (ECOG) performance status of 0 or 1 , histologically confirmed non-squamous NSCLC harboring activating EGFR mutation (exon 19 deletion or L858R mutation on exon 21), and stage IIIb, IV, or recurrent disease that progressed after first-line treatment with EGFR-TKIs. Other detailed inclusion criteria were as follows: at least one measurable lesion by Response Evaluation Criteria in Solid Tumors (RECIST) 1.1; asymptomatic brain metastasis or symptomatic brain metastasis treated with local treatment such as operation, whole brain radiotherapy (WBRT), or stereotactic radiosurgery (SRS); at least 2 weeks after WBRT or palliative radiotherapy (in the case of SRS, treatment delay was not required); adequate organ function; no other previous systemic cytotoxic chemotherapy (adjuvant or neoadjuvant chemotherapy was allowed); and provision of written informed consent. Exclusion criteria included uncontrolled systemic illness such as diabetes, heart failure, unstable angina, hypertension, or arrhythmia; postobstructive pneumonia or uncontrolled serious infection; pregnant or nursing women (women of reproductive potential had to agree to use an effective contraceptive method); uncontrolled symptomatic brain metastasis or presence of a third space that could not be controlled by drainage; prior history of malignancy within 5 years from study entry except for adequately treated basal cell or squamous cell skin cancer, in situ cervical cancer, or well-treated thyroid cancer.

\section{Study design, endpoints, and treatments}

In this multicenter, randomized, open-label, phase II trial, the primary endpoint was to compare PFS of pemetrexed plus cisplatin combination chemotherapy and pemetrexed as a single agent. Secondary endpoints included overall response rate (ORR), OS, safety and toxicity profiles, and health-related quality of life (HRQOL).

Eligible patients were randomly assigned in a 1:1 ratio to a pemetrexed plus cisplatin combination followed by maintenance pemetrexed (PC) arm and a pemetrexed only (P) arm. Block randomization and a non-stratified method were 
used. Patients in the PC arm were treated with four cycles of pemetrexed $500 \mathrm{mg} / \mathrm{m}^{2}$ and cisplatin $70 \mathrm{mg} / \mathrm{m}^{2}$ intravenously, followed by maintenance pemetrexed as a single agent for every 3 weeks until progression of disease (PD). Patients in the $\mathrm{P}$ arm were treated with pemetrexed 500 $\mathrm{mg} / \mathrm{m}^{2}$ monotherapy every 3 weeks until PD. Patients received vitamin $\mathrm{B} 12$, folate, and dexamethasone treatment as premedications for pemetrexed. Dose reductions, delays, and discontinuations due to toxicity were specified by the protocol.

\section{Response and toxicity assessments}

RECIST 1.1 criteria were used to assess the response to treatment by determining PFS and ORR. Tumor assessment by computed tomography was performed at baseline and repeated every other cycle until progression. Other followup assessments including laboratory tests and chest X-ray were repeated every cycle. After progression, patients continued to be followed up for survival every 8-12 weeks until death. Analyses for efficacy and safety were performed with patients receiving at least one dose of any study drug. Toxicity was assessed in accordance with the National Cancer Institute's Common Terminology Criteria for Adverse Events ver. 4.0. The HRQOL was assessed every two cycles using a validated Korean version of EORTC QLQ-C30 ver. 3.0 and
EORTC QLQ-LC13. The QLQ-C30 is composed of five functional scales, three symptom scales, and global health status, and analyses were performed separately according to these domains.

\section{Statistical analysis}

This study was designed as a phase 2 trial, and we calculated the sample size based on the results of previous phase 3 trials $[14,15]$. We assumed that the control arm (P arm) would have a median PFS of 3 months, and we were interested in the experimental arm (PC arm) for further investigation if its median PFS is 6 months or longer. To this end, we needed 85 eligible patients for this study (42-43 patients per arm). And assuming 10\% dropout or ineligibility, approximately 96 randomly assigned patients (48 per arm) were needed for PFS analysis based on (1) exponential PFS models, (2) one-sided alpha $=5 \%$ and power $=90 \%$, (3) a monthly accrual rate of 5-6 patients, and (4) an additional follow-up period of 1 year. The final data analysis was conducted when 70 events of progression were observed. For each arm, PFS and OS were summarized using the Kaplan-Meier method and compared to those of the other arm using the log-rank test. The response and toxicity were summarized using contingency tables. The ORR and incidence rate of toxicity were compared between the two arms using Pearson's chi-square

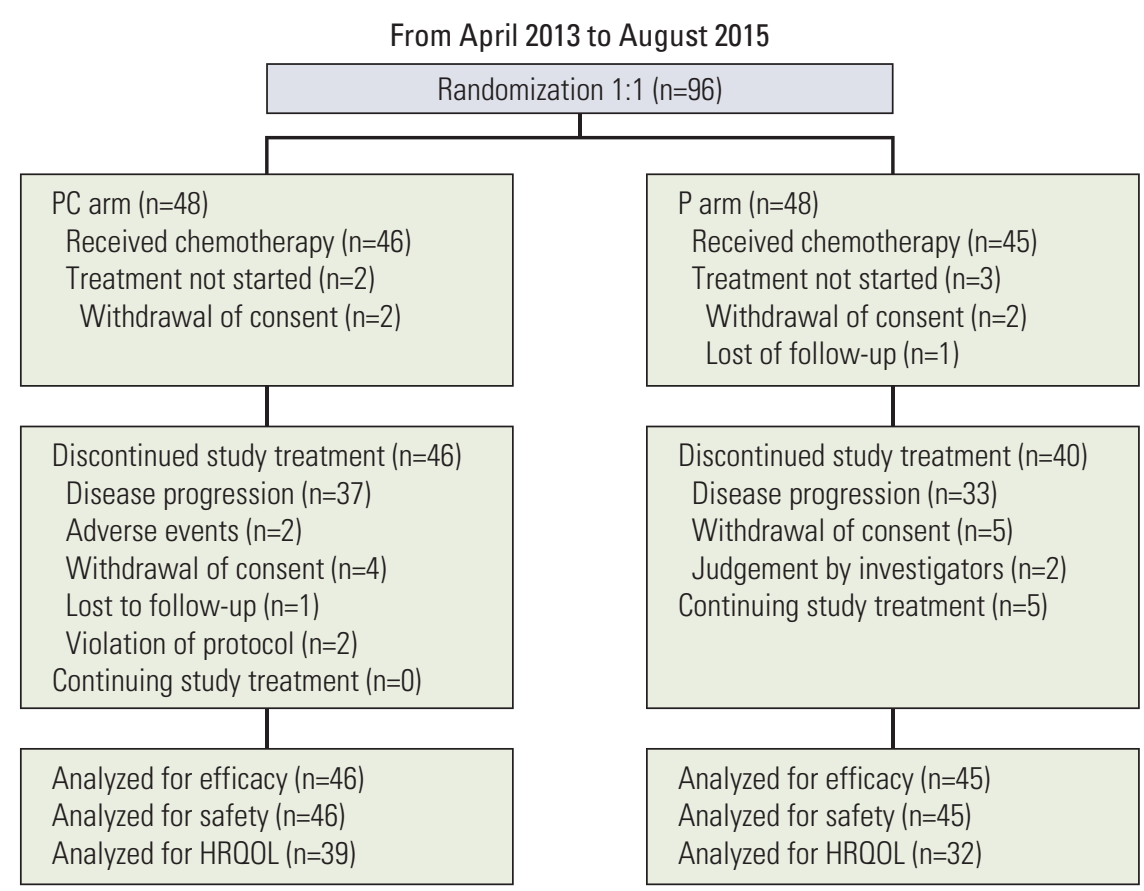

Fig. 1. Patient flow of this study. PC, pemetrexed plus cisplatin combination followed by maintenance pemetrexed; $P$, pemetrexed only; HRQOL, health-related quality of life. 
Table 1. Patient characteristics

\begin{tabular}{|c|c|c|c|c|}
\hline & $P C(n=48)$ & $P(n=48)$ & Total $(n=96)$ & p-value \\
\hline Age (yr) & $60(32-82)$ & $64(31-84)$ & $62(31-84)$ & 0.133 \\
\hline Male sex & $11(23)$ & $15(31)$ & $26(27)$ & 0.358 \\
\hline Smoking, yes & $9(19)$ & $16(33)$ & $25(26)$ & 0.014 \\
\hline \multicolumn{5}{|l|}{ ECOG } \\
\hline 0 & $9(19)$ & $5(10)$ & $14(15)$ & 0.247 \\
\hline 1 & $39(81)$ & $43(90)$ & $82(85)$ & \\
\hline \multicolumn{5}{|l|}{ EGFR mutation } \\
\hline Deletion exon 19 & $32(67)$ & $32(67)$ & $64(67)$ & $>0.999$ \\
\hline L858R & $16(33)$ & $16(33)$ & $32(33)$ & \\
\hline Stage IV & $48(100)$ & $48(100)$ & $96(100)$ & $>0.999$ \\
\hline Recurrence & $1(2)$ & $7(15)$ & $8(8)$ & 0.029 \\
\hline Metastatic & $47(98)$ & $41(85)$ & $88(92)$ & \\
\hline Surgery & $6(13)$ & $9(19)$ & $15(16)$ & 0.399 \\
\hline Radiotherapy & $4(8)$ & $8(17)$ & $12(13)$ & 0.178 \\
\hline
\end{tabular}

Values are presented as median (range) or number (\%). PC, pemetrexed plus cisplatin combination followed by maintenance pemetrexed; P, pemetrexed only; ECOG, Eastern Cooperative Oncology Group; EGFR, epidermal growth factor receptor.

test or Fisher exact test. The HRQOL data were analyzed using Student's t test or Wilcoxon exact test individually or by treatment arm. All tests of treatment effects were conducted at a two-sided alpha level of 0.05 , and all confidence intervals (CIs) were given at a two-sided 95\% level, unless otherwise specified. Analyses were conducted using STATA ver. 14.2 and R 3.2.2 (College Station, TX).

\section{Ethical statement}

All the patients provided written informed consent, and the study was conducted in accordance with the Declaration of Helsinki and Good Clinical Practice guidelines. And the protocol was approved by each participating center's institutional review board.

\section{Results}

\section{Patient characteristics}

From April 2013 to August 2015, a total of 96 patients were recruited from 14 centers in Korea and randomly allocated to PC or P arms. Among 96 randomized patients, 46 in the PC arm and 45 in the P arm were actually treated and formed the target population of analyses for efficacy and safety. Five patients who were not treated with investigational drugs due to withdrawal of consent or loss to follow-up were excluded from the analysis of efficacy and safety (Fig. 1). The clinical data cutoff was October 13, 2016. Patients allocated to the two arms were well balanced for baseline characteristics including age, sex, ECOG performance status, type of EGFR mutation, and stage (Table 1 ). The median age of all patients was 62 years, and $73 \%$ of patients were female. Patients in the $\mathrm{P}$ arm showed a higher rate of smoking than those in the PC arm, but without significance $(p=0.104)$. Most of the patients were ECOG performance status 1 (85\%). In both arms, two-thirds of the patients had exon 19 deletions, and one-third had L858R mutation. All patients were enrolled at stage IV. Fifteen patients $(16 \%)$ underwent curative surgery, and 12 patients $(13 \%)$ had received radiotherapy.

\section{Treatment outcomes and survival}

For the target population of efficacy analyses $(n=91)$, the median number of cycles administered for both PC and $\mathrm{P}$ arms was 6 (range, 1 to 20 cycles for PC and 1 to 43 for P), although the mean number of cycles received was greater for $\mathrm{P}$ than for PC (9.6 vs. 7.1, $\mathrm{p}=0.047)$. The ORR was $34.8 \%$ $(16 / 46)$ in the PC arm and $17.8 \%(8 / 45)$ in the P arm, without statistical significance $(\mathrm{p}=0.066)$. The disease control rate was $78.3 \%(36 / 46)$ in the PC arm and 66.7\% (30/45) in the P arm. During chemotherapy, most patients maintained their initial performance status, although 10 patients $(11 \%)$ experienced temporary worsening.

In a median follow-up of 23.4 months (range, 6.2 to 42.9 months), 70 patients showed disease progression, and 58 deaths occurred. The median PFS was 5.4 months (95\% CI, 
A

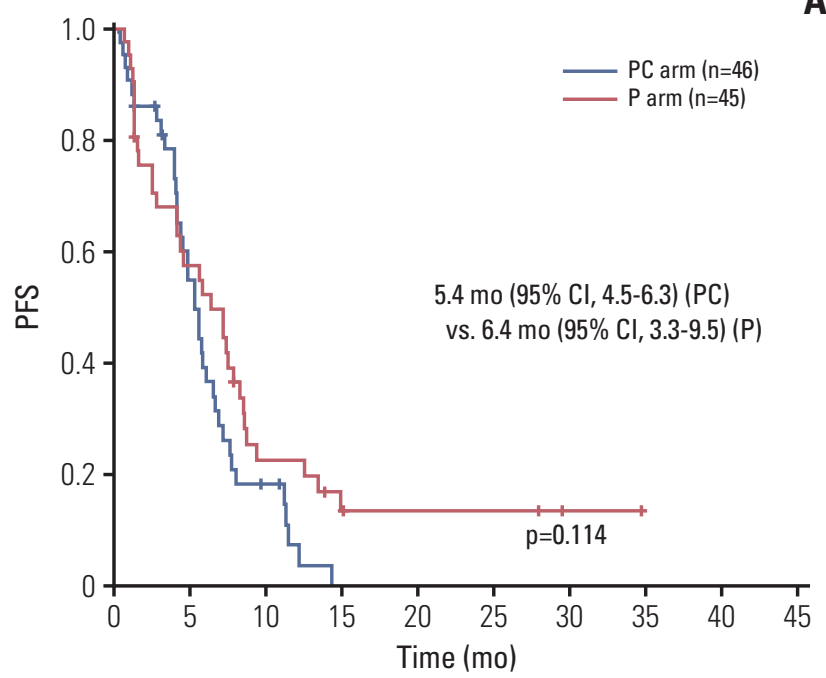

Fig. 2. Kaplan-Meier survival curves of progression-free survival (PFS) (A) and overall survival (OS) (B). PC, pemetrexed plus cisplatin combination followed by maintenance pemetrexed; $\mathrm{P}$, pemetrexed only; $\mathrm{CI}$, confidence interval.

Table 2. Safety and toxicity profiles

\begin{tabular}{|c|c|c|c|c|c|c|c|c|c|}
\hline & \multicolumn{3}{|c|}{$P C(n=46)$} & \multicolumn{3}{|c|}{$P(n=45)$} & \multicolumn{3}{|c|}{$P C+P(n=91)$} \\
\hline & Gr 1-2 & $\mathrm{Gr} \geq 3$ & Total & Gr 1-2 & $\mathrm{Gr} \geq 3$ & Total & Gr 1-2 & $\mathrm{Gr} \geq 3$ & Total \\
\hline Fatigue & 14 & 0 & 14 & 10 & 1 & 11 & 24 & 1 & 25 \\
\hline GI toxicity & 22 & 4 & 26 & 18 & 0 & 18 & 40 & 4 & 44 \\
\hline Bleeding or thrombocytopenia & 1 & 0 & 1 & 1 & 0 & 1 & 2 & 0 & 2 \\
\hline Neutropenia & 1 & 2 & 3 & 0 & 3 & 3 & 1 & 5 & 6 \\
\hline Neurotoxicity & 8 & 0 & 8 & 2 & 0 & 2 & 10 & 0 & 10 \\
\hline Skin and alopecia & 5 & 0 & 5 & 8 & 0 & 8 & 13 & 0 & 13 \\
\hline Myalgia and arthralgia & 2 & 0 & 2 & 4 & 0 & 4 & 6 & 0 & 6 \\
\hline Others $^{\text {a) }}$ & 25 & 8 & 33 & 27 & 5 & 32 & 52 & 13 & 65 \\
\hline
\end{tabular}

PC, pemetrexed plus cisplatin combination followed by maintenance pemetrexed; P, pemetrexed only; Gr, grade; GI, gastrointestinal. ${ }^{a}$ Others include headache, insomnia, dyspnea and pain on other sites than muscles and joints.

4.5 to 6.3 ) in the PC arm and 6.4 months (95\% CI, 3.3 to 5.5) in the $\mathrm{P}$ arm, and there was no significant difference between the two arms ( $\mathrm{p}=0.114$ ) (Fig. 2A). The median OS was 17.9 months (95\% CI, 14.8 to 21.0) and 15.7 months (95\% CI, 13.3 to 18.0) in the PC and P arms, respectively ( $\mathrm{p}=0.787$ ) (Fig. 2B).

\section{Safety and toxicity profiles}

For the target population of efficacy analyses $(n=91), 75$ patients $(82 \%)$ experienced one or more adverse events. The most common adverse event was gastrointestinal toxicities $(\mathrm{n}=44,48.4 \%)$ including nausea, vomiting, diarrhea, and con- stipation. Fatigue was the second most common adverse event ( $\mathrm{n}=25,27.5 \%)$, followed by skin change and / or alopecia $(\mathrm{n}=13,14.3 \%)$, neuropathy $(\mathrm{n}=10,11.0 \%)$, and neutropenia $(n=6,6.6 \%)$. Adverse events $\geq$ grade 3 were observed in 12 patients $(26.1 \%)$ in the PC arm and nine patients $(20.0 \%)$ in the $P$ arm $(\mathrm{p}=0.491)$ (Table 2). Dose reduction (6 vs. 2 patients) and treatment delay (10 vs. 4 patients) were required more often in the PC arm; however, the difference was not significant $(\mathrm{p}=0.267$ for dose reduction, $\mathrm{p}=0.145$ for treatment delay). 

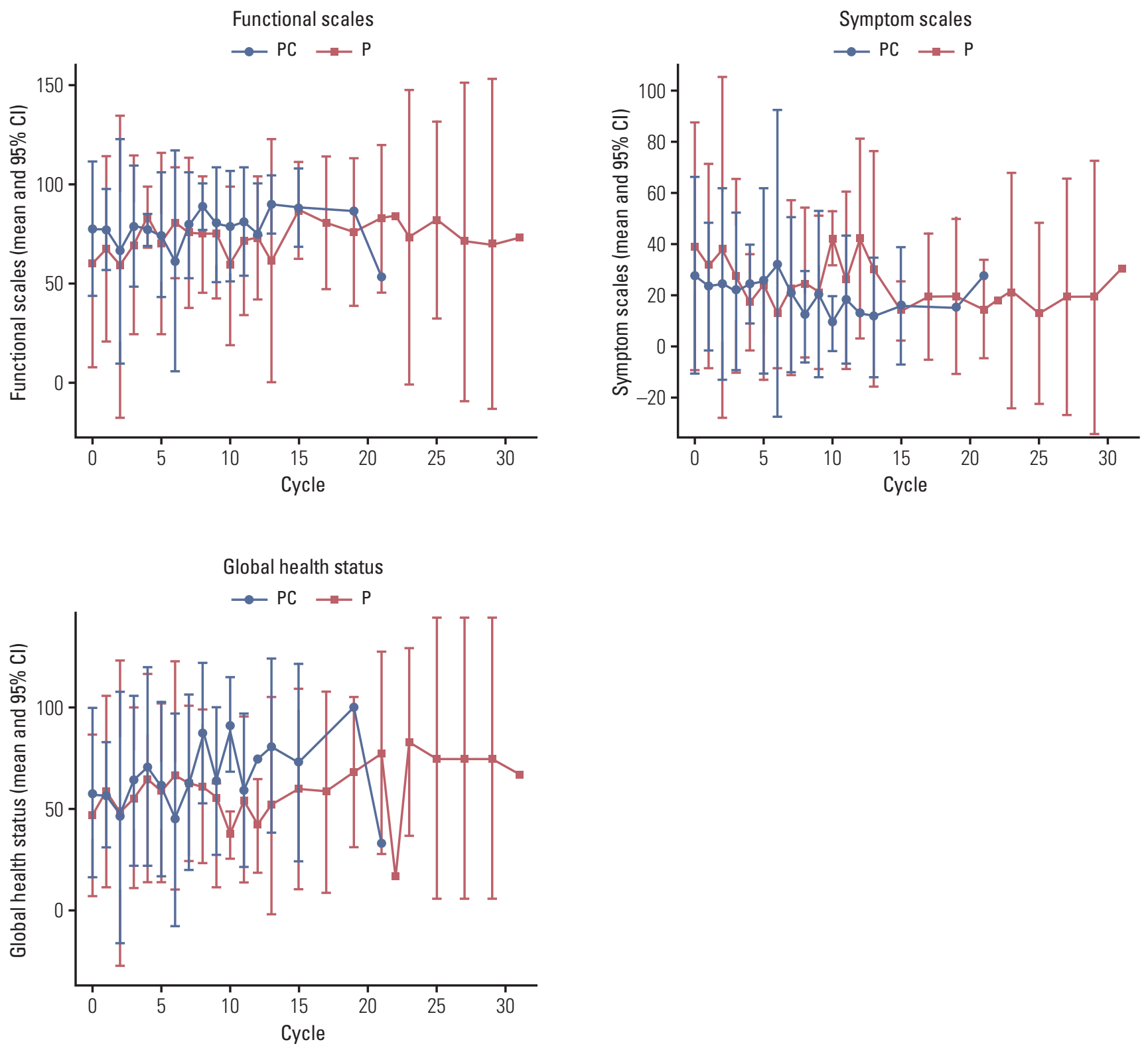

Fig. 3. Health-related quality of life (HRQOL) assessed by EORTC QLQ-C30. PC, pemetrexed plus cisplatin combination followed by maintenance pemetrexed; $\mathrm{P}$, pemetrexed only; $\mathrm{CI}$, confidence interval.

\section{HRQOL}

HRQOL data were obtained from 71 patients (39 in the PC arm and 32 in the $P$ arm) with 385 pairs of EORTC QLQ-C30 and QLO-LC13 questionnaires (185 pairs in the PC arm and 200 in the P arm). For functional scales, patients in the PC arm showed a higher score than patients in the P arm at baseline ( $p<0.05)$; however, no significant differences were observed during follow-up. Likewise, patients in the P arm recorded higher scores for symptom scales than patients in the PC arm at baseline ( $\mathrm{p}<0.05)$, and there was no trend of a difference in statistical significance during chemotherapy. No significant difference was noted in global health status between the two arms (Fig. 3). In assessment of lung cancerassociated symptoms, treatment-related side effects, and pain medication according to questions from the QLQ-LC13, there were no trends of differences between the two arms from baseline through the period of receiving chemotherapy. 


\section{Discussion}

This is the first prospective randomized study to compare pemetrexed plus cisplatin combination therapy with singleagent pemetrexed in patients who have failed first-line EGFR-TKIs. We found that PFS as a primary endpoint was not significantly different between the PC arm and the P arm (5.4 months vs. 6.4 months, $\mathrm{p}=0.114$ ). And ORR also did not make statistically significant difference between two arms ( 34.8 vs. $17.8 \%, \mathrm{p}=0.066$ ). Furthermore, the PC arm did not show improved OS. Intriguingly, quality of life measured by HRQ-OL was similar between the two arms. Our results suggest that single-agent pemetrexed might be another treatment option for patients with failure of first-line EGFR-TKI.

In general, platinum doublet chemotherapy, especially the cisplatin/pemetrexed combination, has been considered standard treatment in patients with failure of targeted agents such as EGFR-TKIs or anaplastic lymphoma kinase inhibitors as first-line therapy because these patients were not exposed to cytotoxic chemotherapy. Single-agent pemetrexed is considered only for patients with poor performance or old age. Accordingly, in the IMPRESS study, a randomized phase III trial comparing gefitinib/chemotherapy and chemotherapy in EGFR mutation-positive NSCLC resistant to first-line gefitinib, the cisplatin plus pemetrexed combination was used as the control arm [9]. A small-sized retrospective study reported prolonged PFS of pemetrexed singlet therapy comparing with nonpemetrexed-based platinum doublet after failure of first-line EGFR-TKIs [16]. Most recently, however, two studies published data indicating the efficacy of pemetrexed-based combination chemotherapy as second-line treatment after development of acquired resistance to firstline EGFR-TKIs $[17,18]$. These studies mutually emphasized the superiority of pemetrexed-containing regimens and combination regimens with platinum in a retrospective analysis. Our previous retrospective study also showed a trend favoring platinum/pemetrexed compared with single-agent pemetrexed in terms of ORR and PFS, which is inconsistent with the results of the current study.

Pemetrexed is a multi-target anti-folate agent and exerts its antitumoral effect mainly by inhibiting the thymidylate synthase (TS) enzyme. Several retrospective and prospective studies have reported an association between lower TS levels and better clinical outcomes for pemetrexed-based chemotherapy $[15,19,20]$. We did not analyze TS level in this study; however, considering that 5.4 months and 6.4 months of PFS in the PC and P arms, respectively, the TS level might be different between two arms. Therefore, additional analysis for the impact of TS expression on the efficacy of pemetrexed after EGFR-TKIs should be performed. Actually, three patients in the $\mathrm{P}$ arm experienced long-term PFS around 30 months (from 28 to 35 months) and are still ongoing. Further molecular analysis for these long-term responders to pemetrexed monotherapy are warranted. Considering the results of pemetrexed plus platinum therapy for NSCLC patients with failure of EGFR-TKIs in both unselected and T790Mpositive populations $[9,10]$, the PFS of 5.4 months and 6.4 months in the PC and P arms in this study would be quite acceptable. Even though no significant differences were observed in PFS and OS between the two arms, PC combination therapy showed a relatively higher response rate and might be a reasonable option for patients who require rapid reduction of tumor burden.

Overall, the incidence of serious adverse events was similar between PC and P arms. Dose reduction and treatment delay were required more often in the PC arm, although the difference was not significant. Furthermore, quality of life assessed by EORTC QLQ-C30 and LC-13 did not show any difference between the two arms. These results might imply that both regimens are equally applicable and tolerable to patients whose disease progresses after EGFR-TKIs.

This study has several limitations. Repeat biopsy to evaluate the resistance mechanism, including T790M, was not performed. It has been reported that patients with the T790M resistance mutation experience an indolent clinical course [21-23]. Even though the two arms were well balanced in terms of patient characteristics, genetic heterogeneity might affect the clinical outcomes. Recently, a third-generation EGFR-TKI, osimertinib, has been approved in patients with T790M resistance mutation [24,25]. And another third-generation EGFR-TKI, olmutinib, received approval only in South Korea for the treatment of patients with T790M mutation-positive NSCLC [26]. Given that systemic chemotherapy is considered standard treatment in T790M-negative patients, it remains undetermined whether the cisplatin plus pemetrexed combination is better than single-agent pemetrexed. Further studies are warranted for this subgroup of the patient population.

In conclusion, pemetrexed plus cisplatin combination treatment and pemetrexed monotherapy might be both effective and well tolerated in EGFR mutation-positive patients who show disease progression on first-line EGFRTKIs. These conventional regimens might be considered as treatment options.

\section{Conflicts of Interest}

This study was supported in part by Lilly, Korea. However, the funder had no role in the study design, data collection and analysis, decision to publish, or preparation of this manuscript. 


\section{Acknowledgments}

This study was supported by a grant from the National R\&D Program for Cancer Control, Ministry of Health and Welfare, Republic of Korea (grant number: 1420260).

\section{Author Details}

1Division of Hematology and Oncology, Department of Medicine, Samsung Medical Center, Sungkyunkwan University School of Medicine, Seoul, ${ }^{2}$ Division of Hematology and Oncology, Department of Internal Medicine, Gachon University Gil Medical Center, Gachon University College of Medicine, Incheon, ${ }^{3}$ Division of Hematology-Oncology, Department of Internal Medicine, Inha University Hospital, Inha University School of Medicine, Incheon ${ }^{4}$ Division of Medical Oncology, Department of Internal Medicine, Chungbuk National University Hospital, Chungbuk National University College of Medicine, Cheongju, ${ }^{5}$ Department of Hematology/Oncology, Keimyung Unversity Dongsan Hospital, Daegu, ${ }^{6}$ Department of Internal Medicine, Seoul National University Boramae Medical Center, Seoul, 'Division of Hematology and Oncology,
Department of Internal Medicine, Dongnam Institute of Radiological and Medical Sciences, Busan, ${ }^{8}$ Division of Medical Oncology, Department of Internal Medicine, Yonsei Cancer Center, Yonsei University College of Medicine, Seoul, ${ }^{9}$ Division of HematologyOncology, Department of Internal Medicine, Gyeongsang Institute of Health Sciences, Gyeongsang National University College of Medicine and Gyeongsang National University Changwon Hospital, Changwon, ${ }^{10}$ Department of Internal Medicine, Gangneung Asan Hospital, University of Ulsan College of Medicine, Gangneung, ${ }^{11}$ Division of Hematology and Oncology, Department of Internal Medicine, National Medical Center, Seoul, ${ }^{12}$ Division of Hematology/Oncology, Department of Internal Medicine, Chungnam National University Hospital, Chungnam National University School of Medicine, Daejeon, ${ }^{13}$ Division of Medical Oncology, Department of Internal Medicine, Seoul St. Mary's Hospital, College of Medicine, The Catholic University of Korea, Seoul, ${ }^{14}$ Division of Medical Oncology and Hematology, Department of Internal Medicine, Kyung Hee University Hospital, Seoul, ${ }^{15}$ Division of Hematology and Oncology, Department of Internal Medicine, Inje University Paik Hospital, Busan, Korea, ${ }^{16}$ Department of Biostatistics and Bioinformatics, Duke University, Durham, NC, USA

\section{References}

1. Zhou W, Christiani DC. East meets West: ethnic differences in epidemiology and clinical behaviors of lung cancer between East Asians and Caucasians. Chin J Cancer. 2011;30:287-92.

2. Zhang YL, Yuan JQ, Wang KF, Fu XH, Han XR, Threapleton $\mathrm{D}$, et al. The prevalence of EGFR mutation in patients with non-small cell lung cancer: a systematic review and metaanalysis. Oncotarget. 2016;7:78985-93.

3. Mok TS, Wu YL, Thongprasert S, Yang CH, Chu DT, Saijo N, et al. Gefitinib or carboplatin-paclitaxel in pulmonary adenocarcinoma. N Engl J Med. 2009;361:947-57.

4. Zhou C, Wu YL, Chen G, Feng J, Liu XQ, Wang C, et al. Erlotinib versus chemotherapy as first-line treatment for patients with advanced EGFR mutation-positive non-smallcell lung cancer (OPTIMAL, CTONG-0802): a multicentre, open-label, randomised, phase 3 study. Lancet Oncol. 2011;12: $735-42$.

5. Sequist LV, Yang JC, Yamamoto N, O'Byrne K, Hirsh V, Mok $\mathrm{T}$, et al. Phase III study of afatinib or cisplatin plus pemetrexed in patients with metastatic lung adenocarcinoma with EGFR mutations. J Clin Oncol. 2013;31:3327-34.

6. Pao W, Miller VA, Politi KA, Riely GJ, Somwar R, Zakowski $\mathrm{MF}$, et al. Acquired resistance of lung adenocarcinomas to gefitinib or erlotinib is associated with a second mutation in the EGFR kinase domain. PLoS Med. 2005;2:e73.

7. Onitsuka T, Uramoto H, Nose N, Takenoyama M, Hanagiri T, Sugio K, et al. Acquired resistance to gefitinib: the contribution of mechanisms other than the T790M, MET, and HGF status. Lung Cancer. 2010;68:198-203.

8. Gainor JF, Shaw AT. Emerging paradigms in the development of resistance to tyrosine kinase inhibitors in lung cancer. J Clin Oncol. 2013;31:3987-96.

9. Soria JC, Wu YL, Nakagawa K, Kim SW, Yang JJ, Ahn MJ, et al. Gefitinib plus chemotherapy versus placebo plus chemotherapy in EGFR-mutation-positive non-small-cell lung cancer after progression on first-line gefitinib (IMPRESS): a phase 3 randomised trial. Lancet Oncol. 2015;16:990-8.

10. Mok TS, Wu YL, Ahn MJ, Garassino MC, Kim HR, Ramalingam SS, et al. Osimertinib or platinum-pemetrexed in EGFR T790M-positive lung cancer. N Engl J Med. 2017;376:629-40.

11. D'Addario G, Rauch D, Stupp R, Pless M, Stahel R, Mach N, et al. Multicenter phase II trial of gefitinib first-line therapy followed by chemotherapy in advanced non-small-cell lung cancer (NSCLC): SAKK protocol 19/03. Ann Oncol. 2008;19: $739-45$.

12. Wu JY, Shih JY, Yang CH, Chen KY, Ho CC, Yu CJ, et al. Second-line treatments after first-line gefitinib therapy in advanced nonsmall cell lung cancer. Int J Cancer. 2010;126:24755.

13. Lee SJ, Sun JM, Lee SH, Ahn JS, Park K, Ahn MJ. Pemetrexed plus platinum versus pemetrexed alone in non-small cell lung cancer patients who have progressed after first-line EGFR TKIs. Lung Cancer. 2015;90:261-6. 
14. Paz-Ares LG, de Marinis F, Dediu M, Thomas M, Pujol JL, Bidoli $P$, et al. PARAMOUNT: Final overall survival results of the phase III study of maintenance pemetrexed versus placebo immediately after induction treatment with pemetrexed plus cisplatin for advanced nonsquamous non-small-cell lung cancer. J Clin Oncol. 2013;31:2895-902.

15. Sun JM, Ahn JS, Jung SH, Sun J, Ha SY, Han J, et al. Pemetrexed plus cisplatin versus gemcitabine plus cisplatin according to thymidylate synthase expression in nonsquamous non-small-cell lung cancer: a biomarker-stratified randomized phase II trial. J Clin Oncol. 2015;33:2450-6.

16. Park S, Keam B, Kim SH, Kim KH, Kim YJ, Kim JS, et al. Pemetrexed singlet versus nonpemetrexed-based platinum doublet as second-line chemotherapy after first-line epidermal growth factor receptor (EGFR) tyrosine kinase inhibitor failure in nonsmall cell lung cancer patients with EGFR mutations. Cancer Res Treat. 2015;47:630-7.

17. Tseng YH, Hung HY, Sung YC, Tseng YC, Lee YC, WhangPeng J, et al. Efficacy of chemotherapy in epidermal growth factor receptor (EGFR) mutated metastatic pulmonary adenocarcinoma patients who had acquired resistance to first-line EGFR tyrosine kinase inhibitor (TKI). J Chemother. 2016;28: 50-8.

18. Yang CJ, Tsai MJ, Hung JY, Liu TC, Chou SH, Lee JY, et al. Pemetrexed had significantly better clinical efficacy in patients with stage IV lung adenocarcinoma with susceptible EGFR mutations receiving platinum-based chemotherapy after developing resistance to the first-line gefitinib treatment. Onco Targets Ther. 2016;9:1579-87.

19. Sun JM, Han J, Ahn JS, Park K, Ahn MJ. Significance of thymidylate synthase and thyroid transcription factor 1 expression in patients with nonsquamous non-small cell lung cancer treated with pemetrexed-based chemotherapy. J Thorac Oncol.
2011;6:1392-9.

20. Nicolson MC, Fennell DA, Ferry D, O'Byrne K, Shah R, Potter $\mathrm{V}$, et al. Thymidylate synthase expression and outcome of patients receiving pemetrexed for advanced nonsquamous non-small-cell lung cancer in a prospective blinded assessment phase II clinical trial. J Thorac Oncol. 2013;8:930-9.

21. Oxnard GR, Arcila ME, Sima CS, Riely GJ, Chmielecki J, Kris $\mathrm{MG}$, et al. Acquired resistance to EGFR tyrosine kinase inhibitors in EGFR-mutant lung cancer: distinct natural history of patients with tumors harboring the T790M mutation. Clin Cancer Res. 2011;17:1616-22.

22. Hata A, Katakami N, Yoshioka H, Takeshita J, Tanaka K, Nanjo S, et al. Rebiopsy of non-small cell lung cancer patients with acquired resistance to epidermal growth factor receptortyrosine kinase inhibitor: Comparison between T790M mutation-positive and mutation-negative populations. Cancer. 2013;119:4325-32.

23. Li W, Ren S, Li J, Li A, Fan L, Li X, et al. T790M mutation is associated with better efficacy of treatment beyond progression with EGFR-TKI in advanced NSCLC patients. Lung Cancer. 2014;84:295-300.

24. National Comprehensive Cancer Network. NCCN clinical practice guidelines in oncology, non-small cell lung cancer (version 3.2017) [Internet]. Plymouth Meeting, PA: National Comprehensive Cancer Network; c2016 [cited 2016 Nov 16]. Available from: https: //www.nccn.org/professionals / physician_gls/pdf/nscl.pdf.

25. Khozin S, Weinstock C, Blumenthal GM, Cheng J, He K, Zhuang L, et al. Osimertinib for the treatment of metastatic EGFR T790M mutation-positive non-small cell lung cancer. Clin Cancer Res. 2017;23:2131-5.

26. Kim ES. Olmutinib: first global approval. Drugs. 2016;76: 1153-7. 